\title{
Quantitative analysis of particles, genomes and infectious particles in supernatants of haemorrhagic fever virus cell cultures
}

\author{
Manfred Weidmann ${ }^{1 *}$, Amadou A Sall ${ }^{2}$, Jean-Claude Manuguerra ${ }^{3}$, Lamine Koivogui ${ }^{4}$, Aime Adjami ${ }^{5}$, \\ Faye Fatou Traoré ${ }^{6}$, Kjell-Olof Hedlund ${ }^{7}$, Gunnel Lindegren${ }^{7}$, Ali Mirazimi ${ }^{7}$
}

\begin{abstract}
Information on the replication of viral haemorrhagic fever viruses is not readily available and has never been analysed in a comparative approach. Here, we compared the cell culture growth characteristics of haemorrhagic fever viruses (HFV), of the Arenaviridae, Filoviridae, Bunyaviridae, and Flavivridae virus families by performing quantitative analysis of cell culture supernatants by (i) electron microscopy for the quantification of virus particles, (ii) quantitative real time PCR for the quantification of genomes, and (iii) determination of focus forming units by coating fluorescent antibodies to infected cell monolayers for the quantification of virus infectivity.

The comparative analysis revealed that filovirus and RVFV replication results in a surplus of genomes but varying degrees of packaging efficiency and infectious particles. More efficient replication and packaging was observed for Lassa virus, and Dengue virus resulting in a better yield of infectious particles while, YFV turned out to be most efficient with only 4 particles inducing one FFU. For Crimean-Congo haemorrhagic fever virus (CCHFV) a surplus of empty shells was observed with only one in 24 particles equipped with a genome. The complete particles turned out to be extraordinarily infectious.
\end{abstract}

\section{Background}

Viral haemorrhagic fevers (VHF) are caused by various haemorrhagic fever viruses (HFV), of the Arenaviridae, Filoviridae, Bunyaviridae, and Flavivridae virus families. Only few laboratories specialize in the research on these agents. Basic virological information on these viruses is scant and described exclusively in the frame of case reports and pathological animal models. Although some progress has been achieved concerning the interaction of these viruses with mechanisms of innate immunity [1-9] and nitrite oxide pathways (CCHFV) [7] concise information on their basic virological characteristics is limited. This type of data however is important for biosafety risk assessment purposes. Here, we present a comparative analysis of quantities of HFV in cell culture determined by electron microscopic counting, quantitative real time RT-PCR and focus forming units, which

\footnotetext{
* Correspondence: mweidma@gwdg.de

'Department of Virology, University Medical Center Göttingen, Germany

Full list of author information is available at the end of the article
}

reveal some features of the replication of these viruses that have not been described before.

\section{Methods \\ Virus propagation}

Viral stocks were prepared from Lassa virus (LASV) strain Josiah, Ebola Zaire virus (EBOVZ) strain Mayinga, Ebola Sudan virus (EBOSV) strains Maridi and Boniface, Marburg virus (MARV) strains Ravn, Ozolin and Musoke, Crimean Congo Haemorrhagic fever virus (CCHFV) strain IbAr 10200, Rift Valley fever virus (RVFV) strain ZH 548, Dengue 1 virus (DENV 1) strain 293, Yellow fever virus (YFV) strain Asibi. Confluent Vero cells (ATCC CCL-81) were inoculated with the respective virus and grown between 1 and 10 days in minimal essential medium (MEM) supplemented with penicillin/streptomycin solution, hepes and $2 \%$ heat inactivated foetal calf serum (all Gibco ${ }^{\circledR}$ BRL, Invitrogen, Life Technologies, Paisley, U.K.), at $37^{\circ} \mathrm{C}$. The supernatants with progeny virus were collected and stored at

\section{C) Biomed Central}

(C) 2011 Weidmann et al; licensee BioMed Central Ltd. This is an Open Access article distributed under the terms of the Creative Commons Attribution License (http://creativecommons.org/licenses/by/2.0), which permits unrestricted use, distribution, and reproduction in any medium, provided the original work is properly cited. 
$-80^{\circ} \mathrm{C}$ until use. All handling of live virus was carried out in biosafety (BSL) level 3 or 4 facilities.

\section{Electron microscopy}

The electron microscopy studies were performed using a Philips CM100 electron microscope (Eindhoven, The Netherlands) as previously described [10]. The viral supernatants were fixed in $2.5 \%$ glutaraldehyde (ratio 1:1) for a minimum of 1 hour in BSL3/4 laboratory, before decontamination of the tubes and transfer to BSL2 laboratory following safety instructions. After fixation, virus particles were pelleted on carbon/Formvar-coated 400-mesh copper grids (GilderGrids, Lincolnshire, England). Briefly, $150 \mu \mathrm{l}$ of virus suspension was centrifuged for $10 \mathrm{~min}$ in an Eppendorf $5417 \mathrm{C}$ centrifuge (Hamburg, Germany) with a swing-out rotor at a maximum force of $12,000 \times g$. The grids were placed on the flat bottom of the outer container of Sarstedt microvette CB 300 tubes (Nümbrecht- Rommelsdorf, Germany). Ten grid squares were counted in each case. One particle per square equals a concentration of $1.5 \times$ $10^{5}$ particles per ml. Grids were stained by $2 \%$ tungstophosphoric acid (Merck, Darmstadt, Germany) at pH 6.

\section{Determination of focus-forming units (FFU)}

The viruses were titrated 10-fold from 1:10 to $1: 10^{10}$ in MEM and $100 \mu \mathrm{l}$ of each dilution was transferred to confluent Vero cells in 96 well tissue culture plates, followed by incubation for $24-48$ hours at $37^{\circ} \mathrm{C}$ and $5 \%$ $\mathrm{CO}_{2}$.

Subsequently the supernatants were removed, and the infected cells were washed 3 times with PBS, before fixation by cold acetone $(80 \%)$ in distilled water for $60 \mathrm{~min}$ utes at $-20^{\circ} \mathrm{C}$. After fixation, fluorescent focus-forming assays were performed by incubation of specific antibodies on the infected cells for 30 minutes at $37^{\circ} \mathrm{C}$, followed by incubation with secondary fluorescein-conjugated antibodies for additionally 30 minutes at $37^{\circ} \mathrm{C}$ (table 1). End point titres (FFU) were set at the last dilution giving unequivocal fluorescence.

\section{Quantitative real time PCR}

RNA was isolated in a BSL3/4 laboratory from the viral stocks by treatment with TRIZOL Reagent (ratio 1+3) (Invitrogen) for a minimum of 5 minutes, before decontamination of the tubes and transport to a BSL2 laboratory following the safety instructions. Quantitative real time RT-PCR (qPCR) was performed using published primers and probes and RNA standards for RVFV, YFV, EBOZV, EBOSV, MARV and DENV [11-15] and primers and probes in table 2 for CCHFV and LASV. CCHFV primers were designed in reference to sequences U39455, AF467768, NC_005300, of African CCHFV isolates, the LASV primers were designed in reference to sequences J04324, AF333969 and AF246121. Quantitative RNA standards for CCHFV, LASV were transcribed from the M-segment (CCHFV), the nucleoprotein gene (LASV) and the 3' NTR region (DENV) ligated into pCRII, and evaluated as previously described [13]. qPCR was performed using the QuantiTect Probe RT-PCR Kit (Qiagen, Hilden Germany) on the Light Cycler 2.0 (Roche, Mannheim, Germany), and the following temperature protocol: $\mathrm{RT} 50^{\circ} \mathrm{C}$ for $5 \mathrm{~min}$, activation at

$95^{\circ} \mathrm{C}$ for $15 \mathrm{~min}$, amplification for 45 cycles at $95^{\circ} \mathrm{C}$ for $5 \mathrm{sec}$ and $60^{\circ} \mathrm{C}$ for $15 \mathrm{sec}$. For CCHFV the same protocol with a touchdown in two degree steps from $70^{\circ} \mathrm{C}$ to $64^{\circ} \mathrm{C}$ for 3 cycles each and 33 cycles at $62^{\circ} \mathrm{C}$ was used.

\section{Results}

\section{Real time PCR}

The new qPCR assays for the CCHFV 10200 M-segment, for LASV Josiah-N and DENV 3'NTR showed an analytical sensitivity of 100, 10 and 100 detected RNA molecules and efficiencies $\left(\mathrm{E}=10^{-1 / \text { slope }}-1\right)$ of $0.9,1.3$ and 1.5 respectively.

Table 1 Description of antibodies used for determination of FFU

\begin{tabular}{|c|c|c|}
\hline Virus & Primary antibodies & Secondary antibodies \\
\hline LASV & Mouse serum Anti-Lassa virus (NP), (in-house) & $\begin{array}{l}\text { FITC-conjugated Rabbit Anti-Mouse Immunoglobulins, (DAKO } \\
\text { Cytomation, Denmark) }\end{array}$ \\
\hline $\begin{array}{l}\text { EBOZV/ } \\
\text { EBOSV }\end{array}$ & Mouse serum Anti-Ebola virus (NP), (in-house) & $\begin{array}{l}\text { FITC-conjugated Goat Anti-Mouse IgG, (Jackson ImmunoResearch, } \\
\text { Baltimore, USA) }\end{array}$ \\
\hline MARV & Human patient serum & $\begin{array}{l}\text { FITC-conjugated Goat Anti-Human IgG (FC specific), (Sigma-Aldrich } \\
\text { Ltd., UK) }\end{array}$ \\
\hline CCHFV & Rabbit serum Anti-CCHFV (NP), (in-house) & $\begin{array}{l}\text { FITC-conjugated Goat Anti-Rabbit IgG (H+L), (Jackson } \\
\text { ImmunoResearch, Baltimore, USA) }\end{array}$ \\
\hline RVFV & $\begin{array}{l}\text { Mouse polyclonal antibodies (provided by Michele Bouloy, } \\
\text { Pasteur Institute, Paris). }\end{array}$ & $\begin{array}{l}\text { FITC-conjugated Rabbit Anti-Mouse Immunoglobulins, (DAKO } \\
\text { Cytomation, Denmark) }\end{array}$ \\
\hline DENV 1 & $\begin{array}{l}\text { Mouse monoclonal to Dengue Virus E glycoprotein, (Abcam, } \\
\text { Cambridge, UK) }\end{array}$ & $\begin{array}{l}\text { FITC-conjugated Goat Anti-Mouse IgG, (Jackson ImmunoResearch, } \\
\text { Baltimore, USA) }\end{array}$ \\
\hline YFV & $\begin{array}{l}\text { Mouse Monoclonal to Yellow Fever Virus, (Abcam, Cambridge, } \\
\text { UK) }\end{array}$ & $\begin{array}{l}\text { FITC-conjugated Goat Anti-Mouse IgG, (Jackson ImmunoResearch, } \\
\text { Baltimore, USA) }\end{array}$ \\
\hline
\end{tabular}


Table 2 Primers and probes

\begin{tabular}{llc}
\hline Virus & Oligomer & Sequence $\mathbf{5}$ to $^{\mathbf{3}}$ \\
\hline CCHFV & CCFM FP & TCACCTTAGAGGAGGACACTGAAGG \\
& CCFM RP & CTCTITGAAAGAAAGTGTCATCACAATC \\
& CCHF M LNA & 6FAM - TGGTGTAAGAGAAATC - BBQ \\
LASV & LAS FP & YAACTCTGCATTYTCACATCCC \\
& LAS RP & TGGGMACCTAAGYTCACAGCA \\
& LAS P & 6FAM - ACCACTCCATCTCTCCCAGCC - TMR \\
\hline
\end{tabular}

LNA Nucleotides in bold, IUB code used $\mathrm{Y}$ for $\mathrm{C} / \mathrm{T}$, $\mathrm{M}$ for $\mathrm{A} / \mathrm{C}$, dyes and quenchers used: $6 F A M=, 6-$ Carboxyfluorescein BBQ $=$ Black Berry Quencher, TMR = Tetramethylrhodamin

\section{Determination of virus quantities}

The virus titers were determined from $1 \mathrm{ml}$ virus tissue culture supernatant by (i) electron microscopy (EM), (ii) quantitative real time PCR (qPCR) of RNA genomes extracted from the culture supernatant and (iii) determination of focus forming units (FFU) on cell monolayers infected with the supernatant using fluorescent antibodies.

The viral genome counts were generally from 1 to 2 orders of magnitude ( $\log _{10}$-steps) higher than the particle counts (table 3, column 4). The FFU counts showed much greater differences being up to $5 \log _{10}$-steps lower than the particle counts (table 3, column 5) and 1-6 $\log _{10}$-steps lower than genome counts (table 3, column 6).

The EBOZV and EBOSV strains showed a genome count $+2 \log _{10}$-step higher than the particle count, but much lower FFU counts differing by -3 to $-5 \log _{10}$-steps from the particle count. MARV strains Ravn and Ozolin showed almost equal particle and genome counts, whereas MARV Musoke showed a $-1 \log _{10}$-step reduction in the genome count compared to the particle count. As for EBOZV and EBOSV, the FFU counts of the MARV strains were -3 to $-4 \log _{10}$-steps lower than the particle counts.

The difference between genome and particle count for LASV, RVFV, and DENV was between +1 to $+2 \log _{10^{-}}$ steps. The FFU reduction from the particle count for these viruses ranged between -2 and $-3 \log _{10}$-steps.
YFV was the only virus were particle and FFU count equalled each other topped only by $a+1 \log _{10}$-step genome count and the lowest difference (-1 $\log _{10}$-step) of the FFU count to the genome count.

Apart from YFV, CCHFV showed the lowest level of difference between FFU and particle count (-1 $\log _{10}$-step). In comparison to YFV a $+1 \log _{10}$-step increased genome count over the particle therefore leads to a $-2 \log _{10}$-step reduction of the FFU count to the genome count.

To interpret the data obtained, ratios were formed between all three quantitative data sets. The observed ratios of particles/FFU (table 4, column 1) point out that for LASV, CCHFV and YFV only few particles (1$10^{2}$ ) are associated with one FFU, whereas for all other viruses much higher amounts $\left(10^{3}-10^{5}\right)$ were counted $\left(10^{3}\right.$ for EBOZV, MARV Ravn, MARV Ozolin, RVFV and DENV 1, $10^{4}$ for EBOSV Boniface, MARV Musoke and $10^{5}$ for EBOSV Maridi).

The ratios of genomes per particle and its reciprocal (table 4, column 2 and 3) indicate that most viruses appear to be overproducing genomes only packaging a fraction of genomes into shells to create complete particles. EBOZV, EBOSV, and RVFV seem to produce a surplus in the range of $74-336$ genomes in relation to the shells produced while this surplus is of reduced magnitude for LASV, DENV and YFV (about 15-20). MARV appears to be efficiently packaging every $3^{\text {rd }}$ $5^{\text {th }}$ genome into a particle (table 4 , column 3 ) achieving this by a slight overproduction of shells. The CCHFV machinery produces 23 empty shells for 1 particle packed with a genome. The packed genomes however are highly infectious as indicated by the value of 1 for the ratio of FFU/genomes (table 4 column 4).

\section{Discussion}

The comparative quantitative analysis of virus titers in cell culture using three independent methods revealed some insight into the packaging efficiency of the viruses

Table 3 Quantitative detemination of virus titers

\begin{tabular}{|c|c|c|c|c|c|c|}
\hline Virus & EM particles/ml & PCR genomes/ml & Infectivity FFU/ml & $\begin{array}{c}\log _{10} \text { difference } \\
\text { PCR to EM }\end{array}$ & $\begin{array}{c}\log _{10} \text { difference } \\
\text { FFU to EM }\end{array}$ & $\begin{array}{c}\log _{10} \text { difference } \\
\text { FFU to PCR }\end{array}$ \\
\hline EBOZV Mayinga & $2.5 \times 10^{8}$ & $8.3 \times 10^{10} \pm 3.2 \times 10^{9}$ & $1.0 \times 10^{5}$ & +2 & -3 & -5 \\
\hline EBOSV Maridi & $7.5 \times 10^{8}$ & $8.6 \times 10^{10} \pm 5.4 \times 10^{9}$ & $1.0 \times 10^{3}$ & +2 & -5 & -7 \\
\hline EBOSV Boniface & $8.0 \times 10^{8}$ & $5.9 \times 10^{10} \pm 3.4 \times 10^{9}$ & $1.0 \times 10^{4}$ & +2 & -4 & -6 \\
\hline MARV Ravn & $4.0 \times 10^{8}$ & $1.3 \times 10^{8} \pm 1.8 \times 10^{6}$ & $1.0 \times 10^{5}$ & 0 & -3 & -3 \\
\hline MARV Ozolin & $5.0 \times 10^{9}$ & $1.6 \times 10^{9} \pm 1,1 \times 10^{8}$ & $1.0 \times 10^{6}$ & 0 & -3 & -3 \\
\hline MARV Musoke & $3.0 \times 10^{9}$ & $5.8 \times 10^{8} \pm 2.7 \times 10^{7}$ & $1.0 \times 10^{5}$ & -1 & -4 & -3 \\
\hline LASV Josiah & $1.0 \times 10^{6}$ & $2.2 \times 10^{7} \pm 4.7 \times 10^{6}$ & $1.0 \times 10^{4}$ & +1 & -2 & -3 \\
\hline RVFV ZH548 & $2.5 \times 10^{9}$ & $2.9 \times 10^{11} \pm 4.3 \times 10^{9}$ & $1.0 \times 10^{6}$ & +2 & -3 & -5 \\
\hline DENV-1 231 & $3.0 \times 10^{9}$ & $4.6 \times 10^{10} \pm 4.3 \times 10^{9}$ & $1.0 \times 10^{6}$ & +1 & -3 & -4 \\
\hline YFV Asibi & $4.0 \times 10^{8}$ & $8.2 \times 10^{9} \pm 1.7 \times 10^{8}$ & $1.0 \times 10^{8}$ & +1 & 0 & -1 \\
\hline CCHFV 10200 & $2.5 \times 10^{7}$ & $1.0 \times 10^{6} \pm 6.1 \times 10^{5}$ & $1.0 \times 10^{6}$ & +1 & -1 & -2 \\
\hline
\end{tabular}

EM: calculated from 10 grid squares, qPCR: average and standard deviation of triplicate qPCR results (8 qPCR results for CCHFV), FFU: mean of three titrations. 
Table 4 Selected ratios of quantitative titer results

\begin{tabular}{lcccc}
\hline Virus & $\begin{array}{c}\text { particles/ } \\
\text { FFU }\end{array}$ & $\begin{array}{c}\text { genomes/ } \\
\text { particle }\end{array}$ & $\begin{array}{c}\text { particles/ } \\
\text { genome }\end{array}$ & $\begin{array}{c}\text { genome/ } \\
\text { FFU }\end{array}$ \\
\hline EBOZV & $2.5 \times 10^{3}$ & 336 & 0.00 & $8.3 \times 10^{5}$ \\
Mayinga & & & & \\
EBOSV Maridi & $7.5 \times 10^{5}$ & 115 & 0.01 & $8.6 \times 10^{7}$ \\
EBOSV & $8.0 \times 10^{4}$ & 74 & 0.01 & $5.9 \times 10^{6}$ \\
Boniface & & & & \\
MARV Ravn & $4.0 \times 10^{3}$ & 0.3 & 3.08 & $1.3 \times 10^{3}$ \\
MARV Ozolin & $5.0 \times 10^{3}$ & 0.3 & 2.98 & $1.6 \times 10^{3}$ \\
MARV Musoke & $3.0 \times 10^{4}$ & 0.2 & 5.10 & $5.8 \times 10^{3}$ \\
LASV Josiah & 100 & 22 & 0.05 & $2.2 \times 10^{3}$ \\
RVFV ZH548 & $2.5 \times 10^{3}$ & 119 & 0.01 & $2.9 \times 10^{7}$ \\
DENV 1 231 & $3.0 \times 10^{3}$ & 15 & 0.06 & $4.6 \times 10^{4}$ \\
YFV Asibi & 4 & 21 & 0.05 & 83 \\
CCHFV IbAR & 25 & 0.04 & 24 & 1 \\
10200 & & & & \\
\hline
\end{tabular}

analysed. The viral RNA genomes were quantified from cell culture supernatants, which most certainly contained free RNA genomes from lysed cells for all of the viruses except for CCHFV, which does not induce a cytopathic effect in Vero cells. Nevertheless, there are some prominent differences in the magnitude of genomes and particle production observed.

In the group of the filoviruses there seems to be a clear separation between the growth characteristics of EBOZV and EBOSV on the one hand, and MARV strains on the other hand. While Ebola viruses appear to produce a $2 \log _{10}$-step surplus of genomes over the particles detectable in EM, the particle and genome values for MARV are almost at the same level. Surprisingly in spite of the production surplus of $10^{8}-10^{9}$ particles both filovirus types seem to produce comparatively few infectious particles with FFU counts down by 3 to $4 \log _{10^{-}}$ steps from the amount of particles (and genomes) for MARV and 4 to $5 \log _{10}$-steps down for EBOZV and EBOSV. Due to the overproduction the resulting amounts of FFU are still veritable but it appears that efficient genome packing by MARV (1 genome in 3-5 particles) by producing more shells than genomes does not lead to a high rate of infectious particles, as about 1000 particles are needed to induce one FFU. For EBOZV and EBOSV a 100-fold overproduction of genomes only yields an even lower amount of infectious particles, as $10^{3}-10^{5}$ are needed to induce one FFU. It seems fair to say that at least as observed in cell culture particle and genome production seem to run out of sync resulting in a proportionally low amount of infectious particles.

In experimental animal models for EBOZV doses as low as one plaque-forming unit suffice to cause infection [16]. The amount of particles recorded for one FFU indicate that although particle production is not very efficient indeed the overall high amount of particles present in one plaque guarantee infection.

Cellular growth of RVFV seems comparable to that of EBOZV Mayinga with very high yields of particles $\left(10^{8}\right)$ and genomes $\left(10^{11}\right)$ leading to 1 genome in 119 being packed into a particle of which $10^{3}$ are needed to induce one FFU. In recent experiments sheep were successfully inoculated with RVFV at a dose of $1 \times 10^{5}$ TCID $_{50}$ [17]. $\mathrm{TCID}_{50}$ and FFU are not easy to relate but it seems that there is much scope to determine a much lower infectious dose of RVFV.

The difference between particle count and genome count for LASV is low and reflected in a genome to particle ratio of 22 indicating a good correlation between virus particles and genomes produced. The efficiency of the virus particles and their infectiousness (100/FFU) observed in cell culture is reflected by the fact that animal models can be highly susceptible to lethal infection as for example to $2 \mathrm{PFU}$ for inbred strain 13 guinea pig [18]. A similar efficiency of the LASV particles was observed in LASV virus-infected marmoset tissues, which contained significant amounts of viral RNA comparable with levels of viremia (measured in PFU) [19].

The best yield of correctly packaged particles is obtained by YFV and CCHFV indicating efficient synchronisation of shell and genome production (table 4 , column 4) by these viruses.

DENV packs genomes even more efficiently than LASV and YFV ( 1 in 15 as compared to 1 in 20). The resulting DENV particles are however much less infectious than the YFV particles and about $10^{3}$ are needed to induce one FFU.

YFV presents itself as the most efficient packer, leading to a very high ratio of infectious particles since it is the only virus for which particle count and FFU count are the same. From the ratios formed it appears that a slight overproduction of genomes $\left(1 \log _{10}\right.$ - steps up from the particle count) is conducive to a genome packaging ratio of 1 in 21 of which only 4 are needed to induce one FFU. Rhesus monkeys have been successfully infected with $7 \times 10^{2}$ plaque forming units (PFU) determined on vero E6 cells [20]. Our results show that the YFV particles indeed are highly infectious and that the infectious dose needed for animal models might be even below the dose used.

CCHFV does not induce a CPE in vero cells and therefore additional viral RNA genomes released by cell lysis do not contribute to the genome count. Therefore the assembly modalities of the infectious CCHFV particles appear reverse to those of the other viruses, as only every 24th particle will actually contain a genome leading to a surplus of empty shells. The correctly assembled particles however are highly infectious as each of them induces a FFU. In the EM analysis 
malformed CCHFV particles were noticed (see Figure 1). Defective interfering particles (DIP) have been described for almost all types of DNA and RNA viruses. DIP have an influence on the viable virus yields (measured in PFU) in cell culture. Particular ratios of DIP to viable virus are conducive to good or bad yields of progeny virus. The mathematical models for this relationship have been extended recently [21]. The extraordinary infectious CCHFV particles (1 genome/FFU) indicate that the malformed CCHFV particles observed might be DIP driving this type of yield.

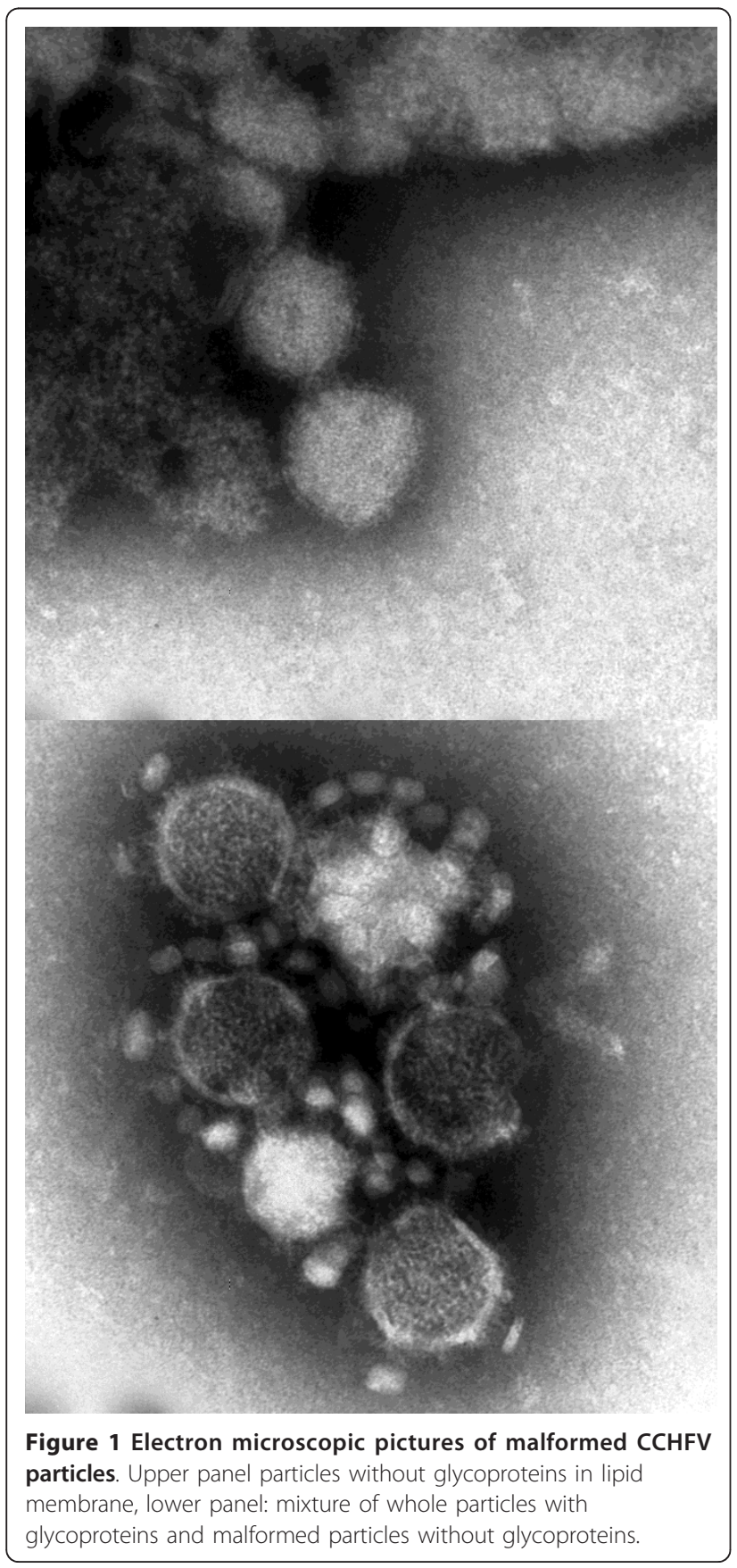

A recent study showed that 10 FFU sufficed to kill IFNAR $^{-1-}$ mice lacking the IFN receptor. These mice do not develop an innate immune response and therefore develop acute disease which makes them a good model for CCHFV infection and disease [22]. These results confirm the extraordinary infectiousness of the CCHFV particles observed in our study.

\section{Conclusion}

The analysed quantitative virus titers indicate that YFV and CCHFV virus are the most efficient in producing infectious particles. These viruses manage to synchronise genome and particle production in an optimal fashion as opposed for example to the filoviruses with an apparent overflowing and inefficient production of genomes and particles. YFV and CCHFV represent the optimal synchronisation of the replication strategies observed i.e. overproduction of genomes and overproduction of shells respectively.

\section{Acknowledgements}

This work was supported by EU grant FP6-INCO-032180 'Development of rapid field diagnostics for identification, control and management of haemorrhagic fever outbreaks (VHF Diagnostics). We thank Frank T. Hufert and Meik Dilcher for critically reading the manuscript.

\section{Author details}

'Department of Virology, University Medical Center Göttingen, Germany. ${ }^{2}{ }^{\prime}$ Institut Pasteur de Dakar, Senegal. Institut Pasteur, CIBU, Paris, France. ${ }^{4}$ Institute of Medical Microbiology Université de Conakry, Guinea. ${ }^{5}$ Multi Disease Surveillance Centre WHO, Ougadougou, Burkina Faso. ${ }^{6}$ Fondation Merieux Mali, Bamako, Mali. ${ }^{7}$ Center for microbiological preparedness, Swedish Institute for Infectious Disease Control, Solna, Sweden.

\section{Authors' contributions}

MW: performed qPCR drafted manuscript, AS: provided strains, involved in drafting and revising the manuscript, has given final approval of the version to be published.

JCM: involved in drafting and revising the manuscript, has given final approval of the version to be published, LK: revised the manuscript, has given final approval of the version to be published, $A A$ : revised the manuscript, has given final approval of the version to be published, FFT: revised the manuscript, has given final approval of the version to be published, $\mathrm{KOH}$ : performed $\mathrm{EM}$, has given final approval of the version to be published, GL: performed FFU assay, has given final approval of the version to be published, AM: involved in drafting and revising the manuscript, has given final approval of the version to be published.

\section{Competing interests}

The authors declare that they have no competing interests.

Received: 17 February 2011 Accepted: 24 February 2011

Published: 24 February 2011

\section{References}

1. Fan L, Briese T, Lipkin Wl: Z proteins of New World arenaviruses bind RIG-I and interfere with type I interferon induction. J Virol 2010 84:1785-1791.

2. Mohamadzadeh M: Potential factors induced by filoviruses that lead to immune supression. Curr Mol Med 2009, 9:174-185.

3. Le May N, Mansuroglu Z, Leger P, Josse T, Blot G, Billecocq A, Flick R, Jacob Y, Bonnefoy E, Bouloy M: A SAP30 complex inhibits IFN-beta expression in Rift Valley fever virus infected cells. PLOS Pathog 2008 $4: \mathrm{e} 13$ 
4. Hartman AL, Bird BH, Towner JS, Antoniadou ZA, Zaki SR, Nichol ST: Inhibition of IRF-3 activation by VP35 is critical for the high level of virulence of ebola virus. J Virol 2008, 82:2699-2704.

5. Andersson I, Karlberg H, Mousavi-Jazi M, Martinez-Sobrido L, Weber F, Mirazimi A: Crimean-Congo hemorrhagic fever virus delays activation of the innate immune response. J Med Virol 2008, 80:1397-1404.

6. Haasnoot J, de Vries W, Geutjes EJ, Prins M, de Haan P, Berkhout B: The Ebola virus VP35 protein is a suppressor of RNA silencing. PLOS Pathog 2007, 3:e86.

7. Simon M, Falk Kl, Lundkvist A, Mirazimi A: Exogenous nitric oxide inhibits Crimean Congo hemorrhagic fever virus. Virus Res 2006, 120:184-190.

8. Le May N, Dubaele S, Proietti De Santis L, Billecocq A, Bouloy M, Egly JM: TFIIH transcription factor, a target for the Rift Valley hemorrhagic fever virus. Cell 2004, 116:541-550.

9. Billecocq A, Spiegel M, Vialat P, Kohl A, Weber F, Bouloy M, Haller O: NSs protein of Rift Valley fever virus blocks interferon production by inhibiting host gene transcription. J Virol 2004, 78:9798-9806.

10. Hardestam J, Simon M, Hedlund KO, Vaheri A, Klingstrom J, Lundkvist A: Ex vivo stability of the rodent-borne Hantaan virus in comparison to that of arthropod-borne members of the Bunyaviridae family. Appl Environ Microbiol 2007, 73:2547-2551.

11. Weidmann M, Sanchez-Seco MP, Sall AA, Ly PO, Thiongane Y, Lo MM, Schley $H$, Hufert FT: Rapid detection of important human pathogenic Phleboviruses. J Clin Virol 2008, 41:138-142.

12. Weidmann M, Faye $O$, Kranaster $R$, Marx A, Nunes MR, Vasconcelos PF, Hufert FT, Sall AA: Improved LNA probe-based assay for the detection of African and South American yellow fever virus strains. J Clin Virol 2010, 48:187-192.

13. Weidmann M, Muhlberger E, Hufert F: Rapid detection protocol for filoviruses. J Clin Virol 2004, 30:94-99.

14. Weidmann M, Hufert FT, Sall AA: Viral load among patients infected with Marburgvirus in Angola. J Clin Virol 2007, 39:65-66.

15. Wagner D, de With K, Huzly D, Hufert F, Weidmann M, Breisinger S, Eppinger S, Kern WV, Bauer TM: Nosocomial acquisition of dengue. Emerg Infect Dis 2004, 10:1872-1873.

16. Bray M, Davis K, Geisbert T, Schmaljohn C, Huggins J: A mouse model for evaluation of prophylaxis and therapy of Ebola hemorrhagic fever. $J$ Infect Dis 1999, 179(Suppl 1):S248-258.

17. Busquets N, Xavier F, Martin-Folgar R, Lorenzo G, Galindo-Cardiel I, del Val BP, Rivas R, Iglesias J, Rodriguez F, Solanes D, et al: Experimental infection of young adult European breed sheep with Rift Valley fever virus field isolates. Vector Borne Zoonotic Dis 2010, 10:689-696.

18. Jahrling PB, Smith S, Hesse RA, Rhoderick JB: Pathogenesis of Lassa virus infection in guinea pigs. Infect Immun 1982, 37:771-778.

19. Carrion R Jr, Brasky K, Mansfield K, Johnson C, Gonzales M, Ticer A, Lukashevich I, Tardif S, Patterson J: Lassa virus infection in experimentally infected marmosets: liver pathology and immunophenotypic alterations in target tissues. J Virol 2007, 81:6482-6490.

20. Arroyo Jl, Apperson SA, Cropp CB, Marafino BJ Jr, Monath TP, Tesh RB, Shope RE, Garcia-Blanco MA: Effect of human gamma interferon on yellow fever virus infection. Am J Trop Med Hyg 1988, 38:647-650.

21. Thompson KA, Yin J: Population dynamics of an RNA virus and its defective interfering particles in passage cultures. Virol J 2010, 7:257.

22. Bereczky S, Lindegren $G$, Karlberg $H$, Akerstrom S, Klingstrom J, Mirazimi A: Crimean-Congo hemorrhagic fever virus infection is lethal for adult type I interferon receptor-knockout mice. J Gen Virol 2010, 91:1473-1477.

doi:10.1186/1743-422X-8-81

Cite this article as: Weidmann et al:: Quantitative analysis of particles, genomes and infectious particles in supernatants of haemorrhagic fever virus cell cultures. Virology Journal 2011 8:81.

\section{Submit your next manuscript to BioMed Central and take full advantage of:}

- Convenient online submission

- Thorough peer review

- No space constraints or color figure charges

- Immediate publication on acceptance

- Inclusion in PubMed, CAS, Scopus and Google Scholar

- Research which is freely available for redistribution

Submit your manuscript at www.biomedcentral.com/submit
Biomed Central 